\title{
Effect of mixed solvents on polyelectrolyte complexes with salt
}

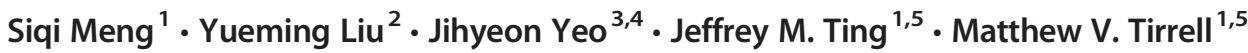 \\ Received: 29 January 2020 / Revised: 9 March 2020 / Accepted: 10 March 2020 / Published online: 8 April 2020 \\ (C) The Author(s) 2020
}

\begin{abstract}
Strongly interacting polyelectrolyte complexes (PECs) are a versatile class of materials whose physical states can be driven from solids into liquids and ultimately into homogenous solution upon salt addition. However, many of these materials can display high stability using common monovalent salts, leading to difficulties in accessing the entire PEC spectrum. Here, the model system, composed of two styrenic polyelectrolytes, required exceptionally high salt to drive phase transition. We term the amount of salt required to drive these transitions salt resistance. In water, the PEC transferred from solid into liquid at $2.5 \mathrm{M} \mathrm{NaBr}$ and never fully dissociated within the studied salt range. We discovered an unconventional approach of weakening salt resistance by switching the solvent to miscible ethylene glycol/water and ethanol/water, allowing us to systematically introduce more hydrophobic constituents. Employing microscopy to determine physical states qualitatively, we found that higher hydrophobicity lowered salt resistance for phase transition and disassembly.
\end{abstract}

Keywords Microscopy (optical, electron, force, fluorescence) $\cdot$ Phase-change materials $\cdot$ Phase separation · Polyelectrolytes · Properties $\cdot$ Optical $\cdot$ Self-assembly $\cdot$ Responsive systems $\cdot$ Polymerization $\cdot$ Living radical

\section{Introduction}

Polyelectrolyte complexes (PECs), the polymer-dense phase formed when two oppositely charged polyelectrolyte solutions are mixed together, [1-6]) are very common in biological and natural systems [7-12]. In addition, they have utility in a wide range of practical applications that are broadly pertinent to overlapping areas in materials science, colloidal science, and engineering, including consumer products, underwater adhesives, and biopharmaceuticals [13-18]. The complexation process is

Matthew V. Tirrell

mtirrell@uchicago.edu

Siqi Meng

siqimeng@uchicago.edu

1 Pritzker School of Molecular Engineering, University of Chicago, Chicago, IL 60637, USA

2 Department of Polymer Science and Engineering, Zhejiang University, Hangzhou 310027, China

3 Department of Chemistry, University of Chicago, Chicago, IL 60637, USA

4 Department of Biochemistry and Molecular Biology, University of Chicago, Chicago, IL 60637, USA

5 Argonne National Laboratory, Lemont, IL 60439, USA generally driven by a favorable gain in entropy upon releasing counterions, forming intrinsic ion pairs and restructuring water molecules around the complex assemblies.

In this mechanism, depending on the chemical and ionic nature of the pair of polyelectrolytes, the physical state of PEC materials can span from glassy solids to low viscosity liquids [19-23]. The phase of the final material has been conventionally hypothesized to be undoubtedly dominated by the nature of polyelectrolyte chain components [18, 24-26]. For instance, $\mathrm{Li}$ et al. have shown that the self-assembly of two charged hydrophilic polypeptides, poly(lysine) and poly(glutamic acid), resulted in a low viscosity liquid in water [27]. The polypeptides have carbonyl and amino groups along the chain backbone that facilitate hydrogen bonding, and the charged moieties can deprotonate depending on $\mathrm{pH}$ conditions. In contrast, the PEC system studied in this present work, composed of poly[(vinylbenzyl) trimethylammonium chloride] (PVBTMA) and poly[sodium 4-styrenesulfonate] (PSS), formed solid precipitates in water (Scheme 1). Here, the aliphatic backbone and aromatic side groups increase the hydrophobicity of the polyelectrolytes, and the trimethylammonium and sulfonate groups are $\mathrm{pH}$-independent. Marras et al. previously compared how the phase behavior of oligonucleotidecontaining complexes depends on the selection of PVBTMA versus poly(lysine) at various degrees of polymerization [14], 
Scheme 1 a Poly[(vinylbenzyl) trimethylammonium chloride] (PVBTMA) chemical structure, $\mathbf{b}$ poly(sodium 4-styrenesulfonate) (PSS) chemical structure, and $\mathbf{c}$ a representative photograph of the solid PVBTMA/PSS polyelectrolyte complex (a)<smiles>CC(C)(C)CC(c1ccc(C[N+](C)(C)Cl)cc1)C(C)(C)C</smiles>

(b)<smiles>CC(C)(C)CC(c1ccc(S(=O)(=O)[O-])cc1)C(C)(C)C</smiles>

(c)

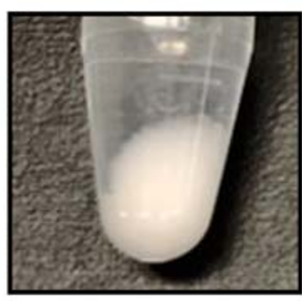

illustrating the importance of molecular level details that are responsible for solid or liquid PEC formation.

In addition to the selection of starting materials, the condition of the external environment also plays a critical role in controlling the physical state of the resultant PECs. For instance, salt is commonly used as a stimulus to break and compensate the ion pairs between oppositely charged chains, and consequently, dissociate the PECs. For strongly interacting PECs that are initially solid precipitates, the addition of salt can transition solids into liquid coacervates and, ultimately, to homogenous solutions [20]. Following this established phenomenon, we employed sodium bromide $(\mathrm{NaBr})$ salt to examine the phase transition of PVBTMA/PSS complexes through its complex/coacervate continuum. A full study of the rheological properties, phase behavior, and structure of this system is underway and remains outside the scope of this current work.

Although salt has been highly investigated as an additive over recent decades, the interplay between salt and solvent in PEC materials has received far less attention. In this study, we attempt to fill this gap by exploring how co-solvents (that introduce a hydrophobic constituent) and salinity together can tune the properties of the PVBTMA/PSS system. Selective binary mixtures of water and organic solvents enable a facile way to progressively modulate PEC behavior. Ethylene glycol and ethanol were deliberately selected as organic solvents due to their miscibility with water (Table 1). We first studied how solvent hydrophobicity alone influenced PEC properties by assembling the complex in salt-free ethylene glycol/water co-solvent conditions. Then we incorporated another dimension and added salt into the system to investigate the mutual effect of solvent and salinity. Optical microscopy was mainly used as the experimental approach to qualitatively map out the phase behaviors of these complexes. This study demonstrates how PECs, as a class of "smart" stimuli-responsive materials, can change their properties and respond to the external environment. We believe the results we present in this study will provide useful new insights into enriching and controlling the properties and functionalities of PECs through controlling the local solvent environment in complex materials.

\section{Materials and methods}

\section{Materials}

The following chemicals were reagent grade and used as received unless otherwise specified: ethanol (HPLC Grade, Millipore Sigma), ethylene glycol (Reagent Plus, $\geq 99 \%$, Millipore Sigma), (vinylbenzyl)trimethylammonium chloride (VBTMA, Sigma, 99\%), poly(styrene sulfonate, sodium salt) (PSS, 201,700 g/mol, Polymer Standards Service), sodium bromide (Fisher Scientific, > 99\%), 4-cyano4-(phenylcarbonothioylthio) pentanoic acid (CPhPA, Millipore Sigma), 2,2'-azobis[2-(2-imidazolin-2yl)propane]dihydrochloride (VA-044, Wako Chemicals, USA), acetic acid (glacial, Sigma, $\geq 99.85 \%$ ), sodium acetate trihydrate (Sigma, $\geq 99 \%$ ), and SnakeSkin dialysis tubing (MWCO 3.5 K, 22 mm, Thermo Scientific). All water used during the experiment was filtered from a Milli-Q water purification system at a resistivity of $18.2 \mathrm{M} \Omega \mathrm{cm}$ at $25^{\circ} \mathrm{C}$. The acetate buffer solution was prepared with $0.1 \mathrm{M}$ acetic acid and $0.1 \mathrm{M}$ sodium acetate trihydrate $(0.1 \mathrm{M})(42 / 158, \mathrm{v} / \mathrm{v})$ at $\mathrm{pH} 5.2$.
Table 1 Physical properties of investigated solvents [28]

\begin{tabular}{lllll}
\hline Solvent & Dielectric constant $\left(\right.$ at $\left.0{ }^{\circ} \mathrm{C}\right)$ & $\begin{array}{l}\text { Surface tension } \\
\left(\text { dyn/cm, at } 25{ }^{\circ} \mathrm{C}\right)\end{array}$ & $\begin{array}{l}\text { Viscosity } \\
\left(\mathrm{mPa} \cdot \mathrm{s} \text {, at } 25{ }^{\circ} \mathrm{C}\right)\end{array}$ & Relative polarity \\
\hline Water & 78.5 & 71.97 & 0.8937 & 1.000 \\
Ethylene glycol & 37.7 & 47.99 & 16.1 & 0.790 \\
Ethanol & 24.6 & 22.39 & 1.04 & 0.654 \\
\hline
\end{tabular}




\section{Polymer synthesis}

The complex system studied here is formed by PVBTMA ${ }_{100}$ and PSS $_{100}$ (Chemical structures are shown in Scheme 1 and the subscripts denote the degree of polymerization). $\mathrm{PSS}_{100}$ was used as received (purchased from Polymer Standards Service). PVBTMA 100 was synthesized with aqueous reversible addition-fragmentation chain transfer (RAFT) polymerization to be approximately symmetric to PSS, based on previous work in our group [29]. Desired amount of VA-044 initiator, VBTMA monomer, and CPhPA chain transfer agent (molar equivalence of 1 to 1000 to 10 , respectively) were added to the acetate buffer solution in a dried $25-\mathrm{mL}$ round bottom flask. The flask was then sealed, degassed with dried nitrogen, and heated at $50^{\circ} \mathrm{C}$ and constant stirring for at least $21 \mathrm{~h}$. The reaction was then cooled to room temperature and opened to air and we obtained the crude pink polymer. The crude polymer was then dialyzed against Milli-Q water for 4 cycles of $8 \mathrm{~h}$ each. Lastly, the samples were lyophilized and we achieved ca. $2 \mathrm{~g}$ polymer in the end.

\section{PEC sample preparation}

PECs were prepared under 1:1 stoichiometric charge-matched conditions between polycation and polyanion. The "as prepared" polymer concentration was fixed at $\sim 1 \mathrm{wt} \%(10 \mathrm{mg} /$ $\mathrm{mL}$ ). Following the protocol of the direct dissolution method [30], we added stock solutions of polycations and polyanions sequentially to a solution with desired amounts of $\mathrm{NaBr}$ stock solution (5 M) and co-solvent of Milli-Q water and ethylene glycol or ethanol. After all the solutions were added, samples were immediately vortexed for at least $30 \mathrm{~s}$.

\section{Optical light microscopy imaging}

To visualize PECs directly at the microscale and determine the phases of these samples, we used optical phase contrast microscopy (Leica DMI 6000B with Leica Application Suite (LAS) image acquisition software, Wetzlar, Germany). PEC samples were first prepared in $1.5 \mathrm{~mL}$ microcentrifuge tubes, and immediately $100 \mu \mathrm{L}$ of the well-mixed samples were transferred into ultra-low attachment 96-well plates (Costar, Corning Inc.). The plates were carefully sealed to prevent water evaporation. Imaging was performed 1 day after sample preparation to guarantee complete phase separation. Using ImageJ software, we later adjusted and enhanced the contrast of the acquired images for clarity.

\section{Thermogravimetric analysis}

After PEC samples were prepared in the $1.5 \mathrm{~mL}$ Eppendorf tubes, they were centrifuged at $4000 \times g$ for $15 \mathrm{~min}$. Then $30 \mu \mathrm{L}$ of the supernatant and around 5-mg complex materials were loaded onto aluminum pans whose weights were measured beforehand. After the samples were transferred, the weights of samples together with pans were recorded again. In this way, the mass of samples on the pans can be calculated. Next, the pans loaded with samples were carefully transferred into a Barnstead Thermolyne Furnace 1400.

The furnace temperature was first set to $200^{\circ} \mathrm{C}$ and held for $2.5 \mathrm{~h}$ to evaporate all the solvents within the samples. The weights of pans with samples were measured again afterwards so that we were able to determine the weight of the evaporated solvents. After that, pans loaded with dried samples were put back into the furnace once again and heated at $600{ }^{\circ} \mathrm{C}$ for $12 \mathrm{~h}$. During this process, all the polymer contents were burned and removed. The weights were recorded for the last time, from which we can calculate the removed polymer mass and the mass of salt remains. For each condition, at least three different repeating samples were measured. Dixon's $Q$ test was performed later to identify and discard outliers.

\section{Results and discussion}

\section{Salt-free PECs in ethylene glycol/water solvent mixtures}

We first prepared PVBTMA/PSS complexes under salt-free conditions and characterized the resultant assembly morphologies with microscopy. Figure 1 shows a gallery of optical images of PECs assembled in increasing co-solvent ratios of ethylene glycol to water. As the ethylene glycol content increases, there was no evident change in the physical appearances of samples, which remained dense, amorphous, and opaque solid aggregates. This suggests that any potential co-solvent effect on the PEC material was not visually detectable on the micron scale. To support this observation, we then conducted thermogravimetric analysis (TGA) to quantify the exact distribution of the total solvent (water and ethylene glycol), polymer, and counterion components within the complex. This thermal technique provides a straightforward way to quantitatively measure the relative weights of the liquid, polymer, and salt in the PEC phase [27]. As shown in Fig. 2, the weight percentage of these three components were invariant to the addition of ethylene glycol in the binary solvent mixture, thus confirming the previous conclusions from the microscopy images that addition of co-solvent does not itself disrupt the complex.

\section{$\mathrm{NaBr}$ doped PECs in ethylene glycol/water solvent mixtures}

Next, we incorporated externally added salt into the PEC assemblies and observed the resultant behavior by microscopy. In the series of PECs in pure water only (i.e., without any ethylene glycol as a co-solvent), a phase transition of this 

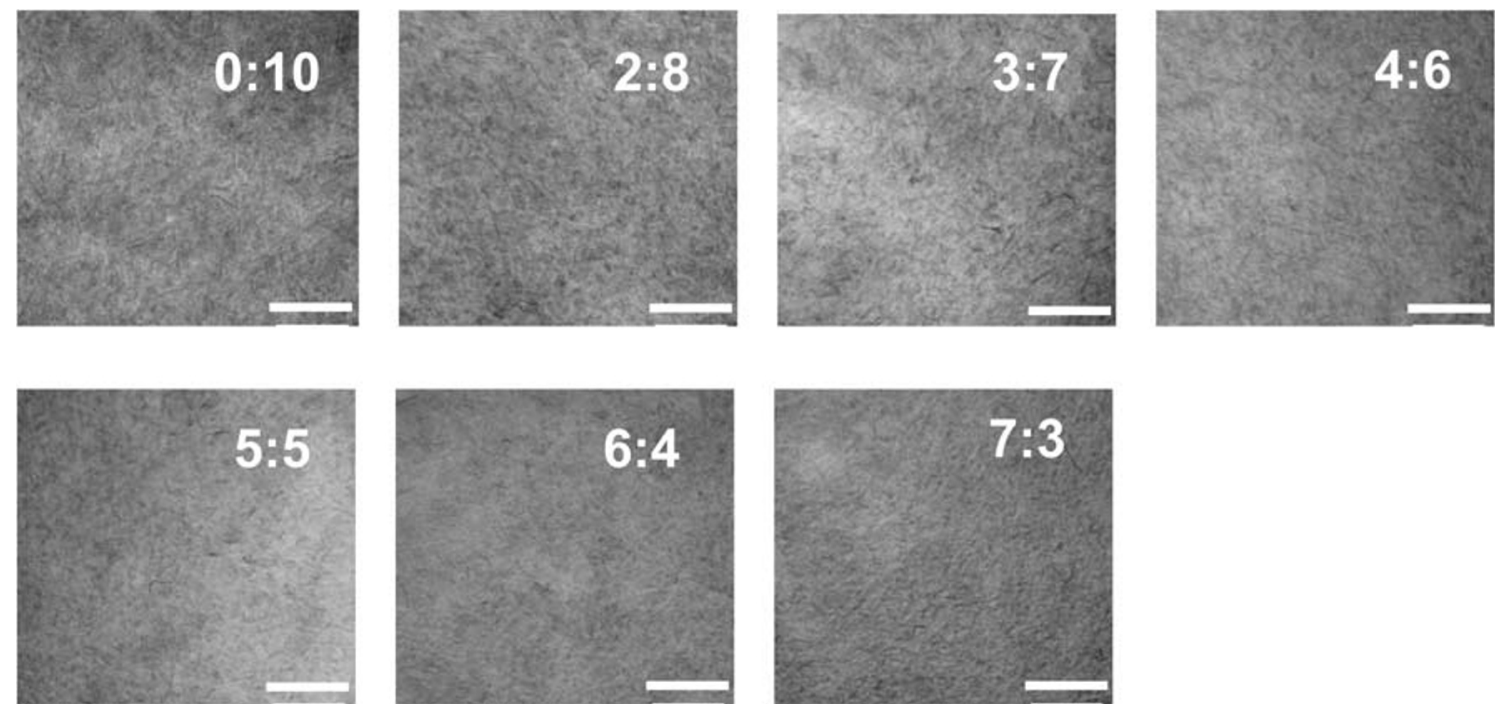

Fig. 1 Optical micrographs of PVBTMA/PSS complexes (without any addition of salt) in ethylene glycol/water mixtures. The white numbers on the top right of each image denote the volumetric ratio of ethylene glycol to water. All scale bars denote $100 \mu \mathrm{m}$

system from solid to liquid was qualitatively mapped by a visual change in their physical appearances in between 2.0 and $2.5 \mathrm{M} \mathrm{NaBr}$, from cloudy and dense aggregates into a fluid-like transparent network (Fig. 3). In other words, the advent of a liquid state can be judged through the emergence of clear structures on microscopy images. This method of determining physical states of this PEC has been confirmed by rheology measurements, which we will discuss in a forthcoming paper.

Although there were no noticeable differences in the PEC as co-solvent effects were introduced in the previous salt-free case, we notice an interesting trend as salt was brought into the PEC system containing ethylene glycol. In the gallery of optical images arranged in Fig. 3, if we navigate vertically from top to bottom, the fraction of ethylene glycol in the co-solvent gradually increases from 0 to 0.7 , and accordingly, the solvent environment becomes more hydrophobic for the PEC phase. As marked by the solid red line, the solid-to-liquid phase transition occurred at lower salt conditions with increasing ethylene glycol content. Additionally, the critical point in salt resistance, where the two-phase PEC system turns into a onephase, homogenous polyelectrolyte solution across the binodal phase boundary, was also lowered as ethylene glycol content increased. This is marked by the solid blue line in Fig. 3. Overall, this set of experiments demonstrates how two orthogonal parameters can be used to transverse the complexcoacervate continuum state space.

\section{$\mathrm{NaBr}$ doped PECs in ethanol/water co-solvent mixtures}

To further expand on the intriguing findings shown in Fig. 3, we prepared otherwise identical PEC samples using ethanol as a co-solvent with water instead of ethylene glycol. As shown in Fig. 4, the same overall pattern was identified by microscopy. Both phase transitions can be carefully tuned as a function of ethanol fraction and $\mathrm{NaBr}$ salt concentration. Furthermore, when directly comparing these two solvent choices, we

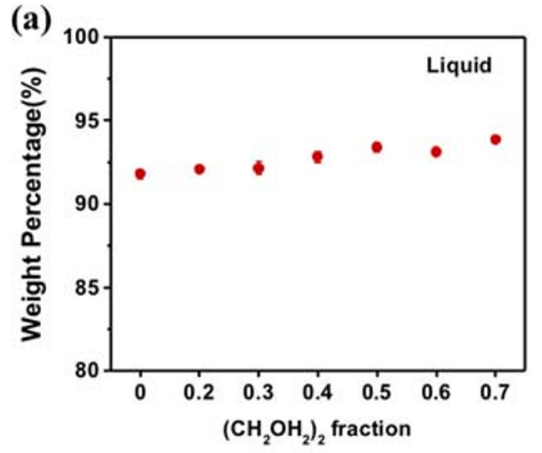

Fig. 2 Weight percentage of the total solvent (a), polymer (b), and counterions (c) within the complex phase of PVBTMA/PSS without the addition of salt as a function of the ethylene glycol fraction within the co-
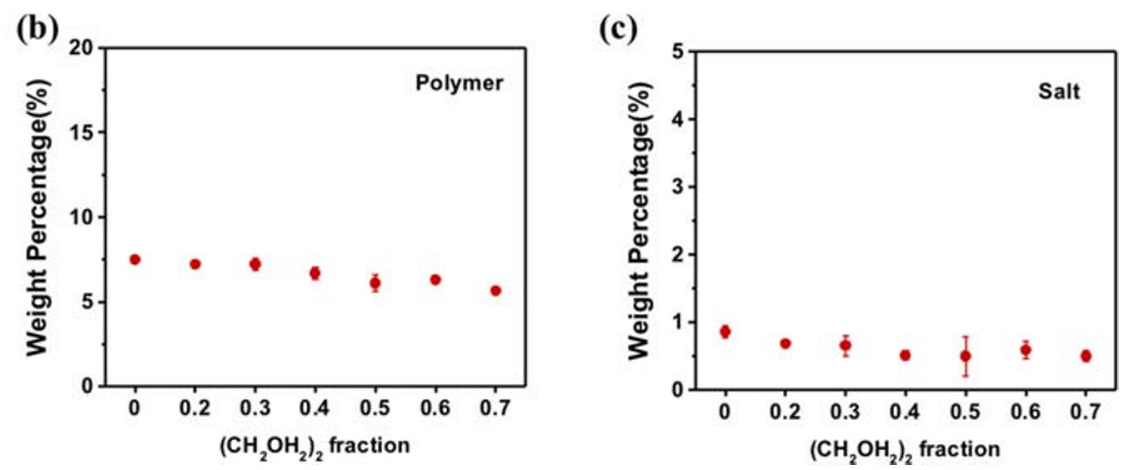

solvent. Circles and vertical error bars represent the mean and standard deviation of at least 3 measurements, respectively 


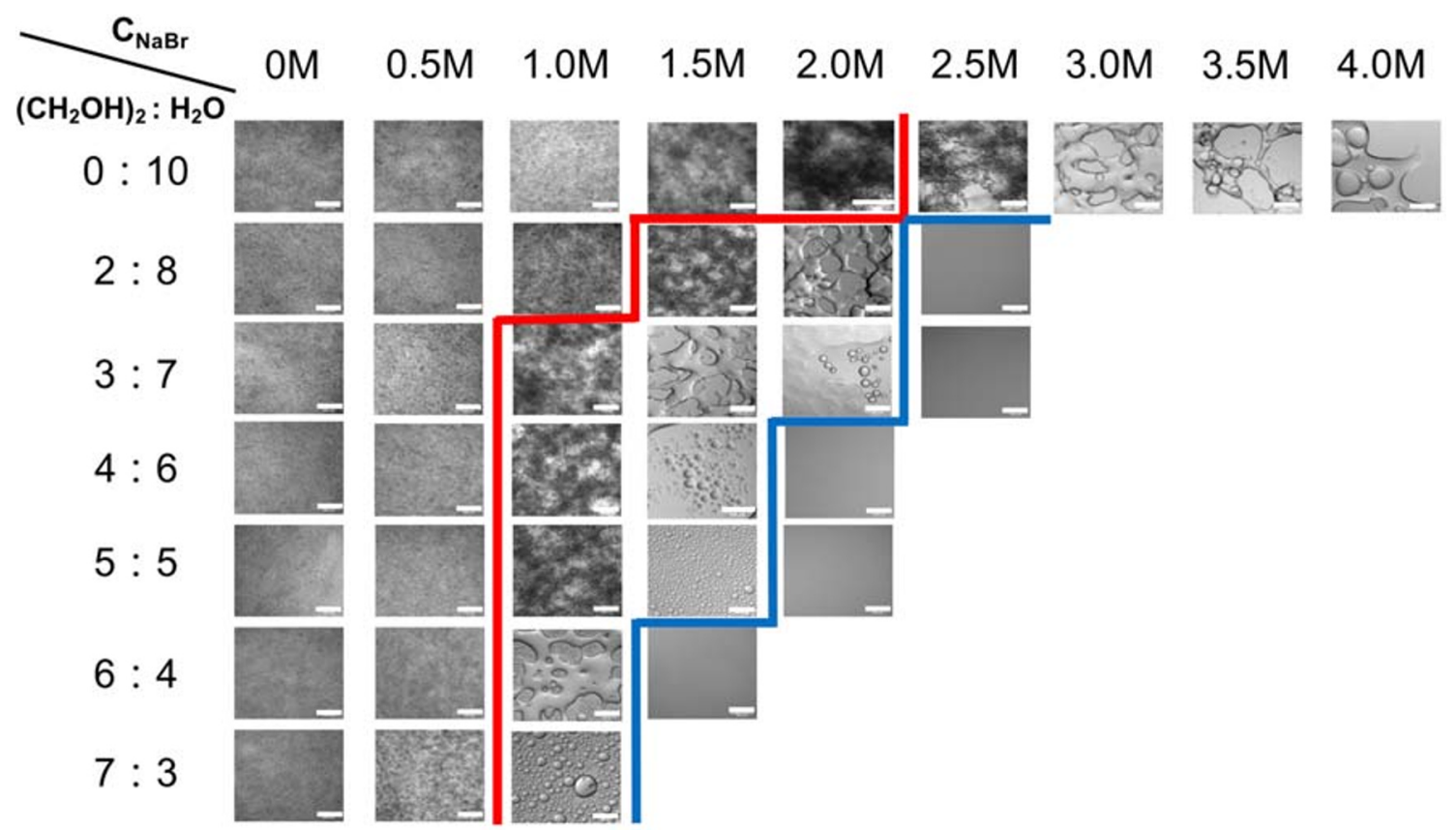

Fig. 3 Optical micrographs of PVBTMA/PSS complexes with 0 to $4.0 \mathrm{M}$ $\mathrm{NaBr}$ (left to right) and in 0:10 to 7:3 volumetric ratios of ethylene glycol/ water mixtures (top to bottom). To guide the eye, the solid red line

noticed that at equivalent ratios of organic solvent to water, both phase transitions with ethylene glycol occurred at higher $\mathrm{NaBr}$ salt concentration levels than with ethanol. For example, at 4:6 organic solvent to water mixtures, for ethylene glycol, solid precipitates converted into liquid coacervates at 0.5 1.0 $\mathrm{M} \mathrm{NaBr}$ and into solution at 1.5-2.0 M NaBr. For ethanol, these transition points were measured to be $0-0.5 \mathrm{M} \mathrm{NaBr}$ and $1.0-1.5 \mathrm{M} \mathrm{NaBr}$, respectively. represents the point where the complexes phase transitions from solid to liquid, and the solid blue line denotes the phase transition from liquid to solution. All scale bars denote $100 \mu \mathrm{m}$

We hypothesize that this difference between ethylene glycol and ethanol is due to the physical properties of these two solvents that influence the associative driving force and salt resistance of this PEC system in water. For instance, the relative polarities of ethanol and ethylene glycol are 0.654 and 0.79 , respectively [28]. Therefore, under the same ratio of organic solvent and water, ethanol/water co-solvent creates a more "hydrophobic" environment for the complex phase,

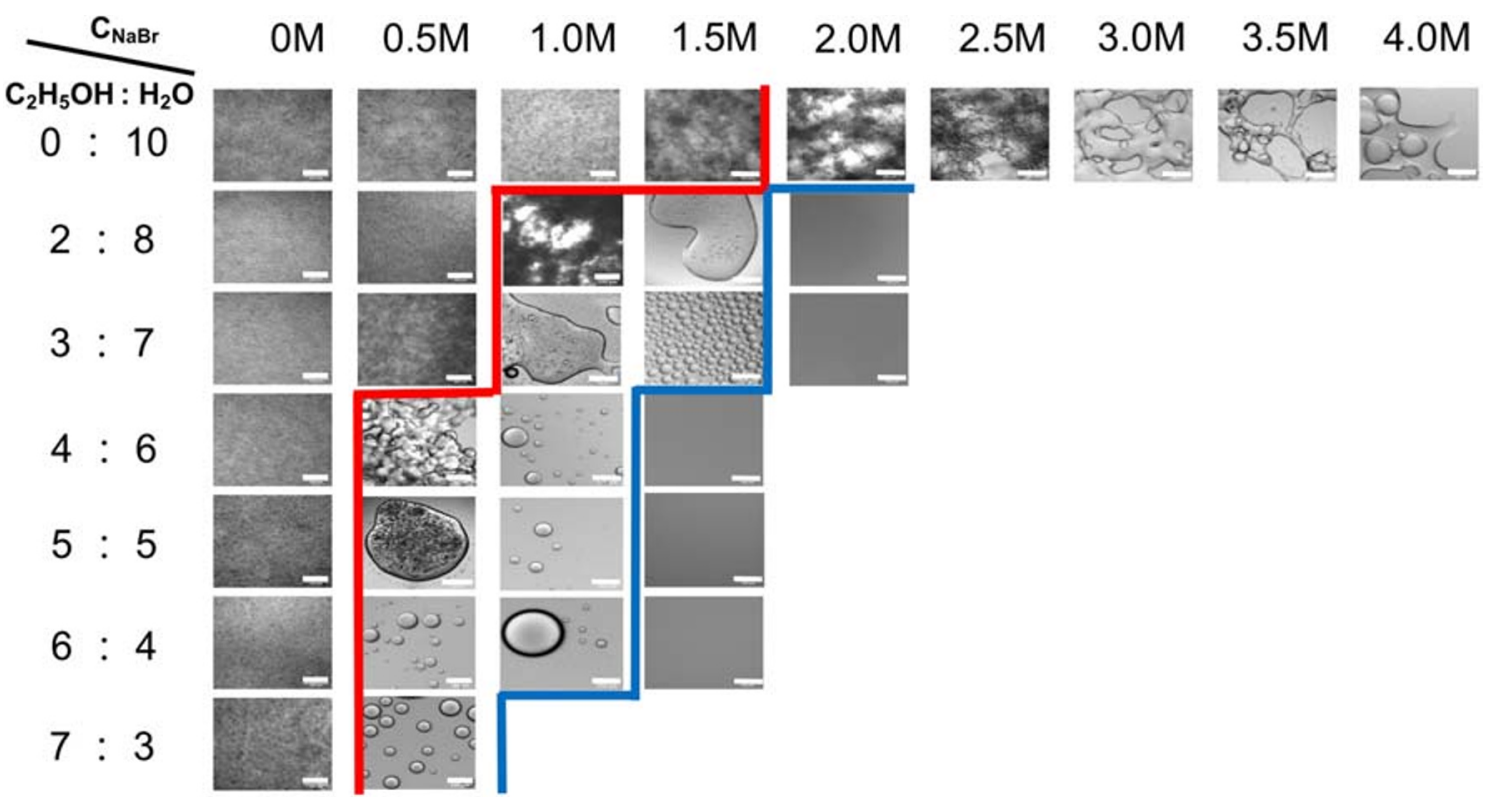

Fig. 4 Optical micrographs of PVBTMA/PSS complexes with 0 to $4.0 \mathrm{M}$ $\mathrm{NaBr}$ (left to right) and in $0: 10$ to 7:3 volumetric ratios of ethanol/water mixtures (top to bottom). To guide the eye, the solid red lines represent the point where the complexes phase transitions from solid to liquid, and the solid blue line denotes the phase transition from liquid to solution. All scale bars denote $100 \mu \mathrm{m}$ 
causing the bulk complex to be less salt resistant. It is worth pointing out here that our observation is the opposite of what has been mentioned in previous publications. Chang et al. [31] have reported a dramatic increase in salt resistance for polypeptide-based coacervates when solvent was switched from water to a more hydrophobic mixture of isopropanol and water. Similarly, Sun et al. [32] have discovered that as the co-solvent became more hydrophobic, there was a slight increase in the salt concentrations needed to drive solid-to-liquid phase transition and complete dissociation of polysaccharide-based PECs. We think this striking difference between our work and previous studies came from the difference in the nature of polymer materials: PVBTMA and PSS are very hydrophobic, while both polypeptides polysaccharides are quite hydrophilic. Nevertheless, there are clearly other subtle factors that govern the effects of solvent on PECs. More rigorous investigations to test the universality of what we have observed in these two limiting cases are underway.

\section{Conclusion}

In summary, by studying comprehensively the assembly of a model PEC system under various co-solvent and salinity conditions, we report a straightforward method of modulating the complex stability and responsivity. To this end, PVBTMA and PSS were ideal since this strong polycation-polyanion pairing resulted in solid precipitates that exhibited high salt resistance in water for systematic co-solvent and salt studies.

When we introduced co-solvents of ethylene glycol and ethanol, both solid-to-liquid and liquid-to-solution phase transitions were tunable and occurred at much lower salt concentrations. This finding suggests that co-solvents can be exploited as a powerful agent to modulate the behavior of strongly interacting PECs upon the addition of salt. Furthermore, by progressively adjusting the co-solvent ratios, we were able to demonstrate a clear correlation between salt resistance and solvent quality: aqueous solvent mixtures can weaken the salt resistance of PEC systems in a systematic manner.

Altogether, this work provides practical insights into enriching our understanding of the phase behavior along the coacervate/complex continuum. Future work in our group will expand on the fundamental mechanisms and molecular details behind this physical behavior for charge-driven assemblies. For amphiphilic surfactants and polymers, solvent quality is a known important factor for both the thermodynamics and kinetics of these self-assembled systems. The extension of this general idea for charged polymers has not yet been fully realized, but careful examination of controlled systems can elucidate important structure-property relationships for the continued integration of PECs into advanced materials applications.
Acknowledgments The authors thank Justin Jureller, $\mathrm{PhD}$, from the James Franck Institute at the University of Chicago for his assistance in thermogravimetric analysis.

Funding information This work was performed under financial assistance award 70NANB19H005 from the US Department of Commerce, National Institute of Standards and Technology as part of the Center for Hierarchical Materials Design (CHiMaD). J.M.T acknowledges financial support from the NIST-CHiMaD Postdoctoral Fellowship.

\section{Compliance with ethical standards}

Conflict of interest The authors declare that they have no conflict of interest.

Open Access This article is licensed under a Creative Commons Attribution 4.0 International License, which permits use, sharing, adaptation, distribution and reproduction in any medium or format, as long as you give appropriate credit to the original author(s) and the source, provide a link to the Creative Commons licence, and indicate if changes were made. The images or other third party material in this article are included in the article's Creative Commons licence, unless indicated otherwise in a credit line to the material. If material is not included in the article's Creative Commons licence and your intended use is not permitted by statutory regulation or exceeds the permitted use, you will need to obtain permission directly from the copyright holder. To view a copy of this licence, visit http://creativecommons.org/licenses/by/4.0/.

\section{References}

1. Michaels AS (1965) Polyelectrolyte complexes. Ind Eng Chem 57: 32-40. https://doi.org/10.1021/ie50670a007

2. Philipp B, Dautzenberg H, Linow KJ, Kötz J, Dawydoff W (1989) Polyelectrolyte complexes - recent developments and open problems. Prog Polym Sci 14:91-172. https://doi.org/10.1016/00796700(89)90018-X

3. Srivastava S, Tirrell MV (2016). Adv Chem Phys 161. https://doi. org/10.1002/9781119324560

4. Veis A (2011) A review of the early development of the thermodynamics of the complex coacervation phase separation. Adv Colloid Interf Sci 167:2-11. https://doi.org/10.1016/j.cis.2011.01.007

5. Overbeek JTG, Voorn MJ (1957) Phase separation in polyelectrolyte solutions. Theory of complex coacervation. J Cell Comp Physiol 49:7-26. https://doi.org/10.1002/jcp.1030490404

6. Priftis D, Xia X, Margossian KO, Perry SL, Leon L, Qin J, De Pablo JJ, Tirrell M (2014) Ternary, tunable polyelectrolyte complex fluids driven by complex coacervation. Macromolecules 47:30763085. https://doi.org/10.1021/ma500245j

7. Zhao H, Sun C, Stewart RJ, Waite JH (2005) Cement proteins of the tube-building polychaete Phragmatopoma californica. J Biol Chem 280:42938-42944. https://doi.org/10.1074/jbc.M508457200

8. Widom J (1998) Structure, dynamics, and function of chromatin in vitro. Annu Rev Biophys Biomol Struct 27:285-327. https:// doi.org/10.1146/annurev.biophys.27.1.285

9. Brangwynne CP, Tompa P, Pappu RV (2015) Polymer physics of intracellular phase transitions 11:899-904. https://doi.org/10.1038/ NPHYS3532

10. Vieregg JR, Lueckheide M, Marciel AB, Leon L, Bologna AJ, Rivera JR, Tirrell MV (2018) Oligonucleotide-peptide complexes: 
phase control by hybridization. J Am Chem Soc 140:1632-1638. https://doi.org/10.1021/jacs.7b03567

11. Smith J, Calidas D, Schmidt H, Lu T, Rasoloson D, Seydoux G (2016) Spatial patterning of P granules by RNA-induced phase separation of the intrinsically-disordered protein MEG-3. Elife 5: 1-18. https://doi.org/10.7554/eLife.21337

12. Brangwynne $C$, Hyman $T$ (2012) In retrospect: the origin of life. Nature 491:524-525. https://doi.org/10.1038/491524a

13. Kuo CH, Leon L, Chung EJ, Huang RT, Sontag TJ, Reardon CA, Getz GS, Tirrell M, Fang Y (2014) Inhibition of atherosclerosispromoting microRNAs via targeted polyelectrolyte complex micelles. J Mater Chem B 2:8142-8153. https://doi.org/10.1039/ c4tb00977k

14. Marras AE, Vieregg JR, Ting JM, Rubien JD, Tirrell MV (2019) Polyelectrolyte complexation of oligonucleotides by charged hydrophobic-neutral hydrophilic block copolymers. Polymers (Basel) 11:83. https://doi.org/10.3390/polym11010083

15. Lueckheide M, Vieregg JR, Bologna AJ, Leon L, Tirrell MV (2018) Structure-property relationships of oligonucleotide polyelectrolyte complex micelles. Nano Lett 18:7111-7117. https://doi.org/10. 1021/acs.nanolett. 8 b03132

16. De Kruif CG, Weinbreck F, De Vries R (2004) Complex coacervation of proteins and anionic polysaccharides. Curr Opin Colloid Interface Sci 9:340-349. https://doi.org/10.1016/j.cocis.2004.09. 006

17. Gouin S (2004) Microencapsulation: industrial appraisal of existing technologies and trends. Trends Food Sci Technol 15:330-347. https://doi.org/10.1016/j.tifs.2003.10.005

18. Perry SL, Leon L, Hoffmann KQ, Kade MJ, Priftis D, Black KA, Wong D, Klein RA, Pierce CF, Margossian KO et al (2015) Chirality-selected phase behaviour in ionic polypeptide complexes. Nat Commun 6:6052. https://doi.org/10.1038/ncomms7052

19. Sadman K, Wang Q, Chen Y, Keshavarz B, Jiang Z, Shull KR (2017) Influence of hydrophobicity on polyelectrolyte complexation. Macromolecules 50:9417-9426. https://doi.org/10.1021/acs. macromol.7b02031

20. Wang, Q.; Schlenoff, J. B. The polyelectrolyte complex/coacervate continuum Macromolecules 2014, 47, 3108-3116. https://doi.org/ 10.1021/ma500500q

21. Liu Y, Momani B, Winter HH, Perry SL (2017) Rheological characterization of liquid-to-solid transitions in bulk polyelectrolyte complexes. Soft Matter 13:7332-7340. https://doi.org/10.1039/ c7sm01285c

22. Tirrell M (2018) Polyelectrolyte complexes: fluid or solid? ACS Cent Sci 4:532-533. https://doi.org/10.1021/acscentsci.8b00284

23. Zhang Y, Batys P, O'Neal JT, Li F, Sammalkorpi M, Lutkenhaus JL (2018) Molecular origin of the glass transition in polyelectrolyte assemblies. ACS Cent Sci 4:638-644. https://doi.org/10.1021/ acscentsci.8b00137

24. Sadman K, Wang Q, Shull KR (2019) Guanidinium can break and form strongly associating ion complexes. ACS Macro Lett 8:117122. https://doi.org/10.1021/acsmacrolett.8b00824

25. Herzog-Arbeitman A, Ting JM, Meng S, Wu H, Tirrell MV (2019) Patterning polyelectrolyte complexes with alternating monomer sequence distributions. ChemRxiv. https://doi.org/10.26434/ chemrxiv.11320439.v1

26. Marciel AB, Srivastava S, Tirrell MV (2018) Structure and rheology of polyelectrolyte complex coacervates. Soft Matter 14:2454 2464. https://doi.org/10.1039/C7SM02041D

27. Li L, Srivastava S, Andreev M, Marciel AB, De Pablo JJ, Tirrell MV (2018) Phase behavior and salt partitioning in polyelectrolyte complex coacervates. Macromolecules 51:2988-2995. https://doi. org/10.1021/acs.macromol.8b00238

28. Lemmon EW, McLinden MO, Friend DG (2017) NIST Chemistry WebBook, NIST Standard Reference Database. https://doi.org/10. 18434/T4D303

29. Ting JM, Wu H, Herzog-Arbeitman A, Srivastava S, Tirrell MV (2018) Synthesis and assembly of designer styrenic diblock polyelectrolytes. ACS Macro Lett 7:726-733. https://doi.org/10.1021/ acsmacrolett.8b00346

30. Wu H, Ting JM, Werba O, Meng S, Tirrell MV (2018) Nonequilibrium phenomena and kinetic pathways in self-assembled polyelectrolyte complexes. J Chem Phys 149:163330-1-10. https://doi.org/10.1063/1.5039621

31. Chang LW, Lytle TK, Radhakrishna M, Madinya JJ, Vélez J, Sing CE, Perry SL (2017) Sequence and entropy-based control of complex coacervates. Nat Commun 8:1273. https://doi.org/10.1038/ s41467-017-01249-1

32. Sun J, Perry SL, Schiffman JD (2019) Electrospinning nanofibers from chitosan/hyaluronic acid complex coacervates. Biomacromolecules 20(11):4191-4198. https://doi.org/10.1021/ acs.biomac. $9 \mathrm{~b} 01072$

Publisher's note Springer Nature remains neutral with regard to jurisdictional claims in published maps and institutional affiliations.

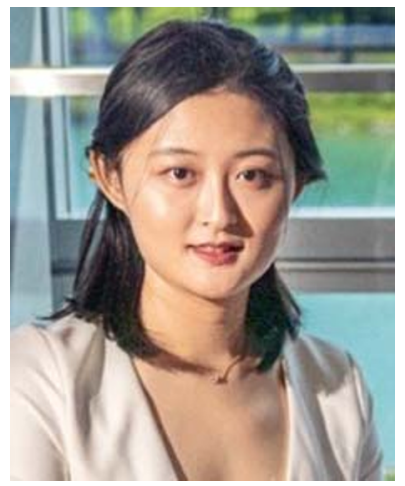

Siqi Meng received her BS degree in Chemical and Biomolecular Engineering at Rice University in 2017. After that, she started her Ph.D. in Pritzker School of Molecular Engineering (PME) at the University of Chicago, where she works with Prof. Matthew Tirrell on investigating and modulating the properties of polyelectrolyte complexes (PECs).

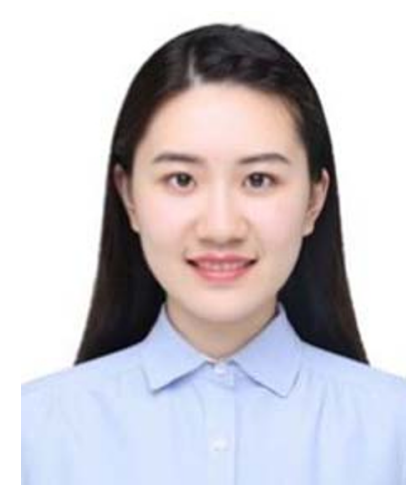

Yueming Liu is now an undergraduate student in Polymer Science and Engineering at Zhejiang University. In summer 2019, she worked with Prof. Matthew Tirrell in Pritzker School of Molecular Engineering (PME) at the University of Chicago, where she studied the properties of polyelectrolyte complexes (PECs). 


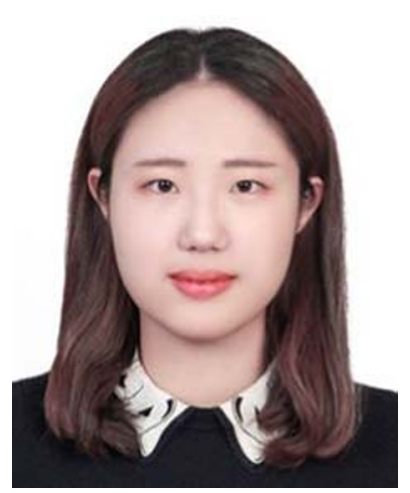

Jihyeon Yeo is a third-year undergraduate student at the University of Chicago, pursuing a B.S. in biological chemistry and a B.A. in chemistry. She is currently a research assistant in Prof. Tirrell's group working on polyelectrolyte complexes and phosphate-selective peptide amphiphile.

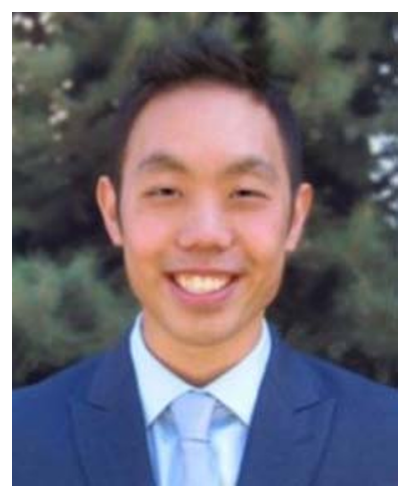

Jeffrey M. Ting received his BS in Chemical Engineering at the University of Texas and his $\mathrm{PhD}$ in Chemical Engineering from the University of Minnesota, where he worked with Frank Bates and Theresa Reineke on synthesizing tunable polymers for oral drug delivery. Jeff was a recipient of the L. E. and D. H. Scriven Fellowship, NSF Graduate Research Fellowship, and Minnesota Doctoral Dissertation Fellowship. His work was recognized by the first annual 2015 AIChE Pharmaceutical Discovery, Development and Manufacturing Student Award. Currently, Dr. Ting is a NIST-CHiMaD Postdoctoral Fellow in Matthew Tirrell's group, as part of the Center for Hierarchical Materials Design (CHiMaD) under the NIST Advanced Materials Center of Excellence program and the Materials Genome Initiative.

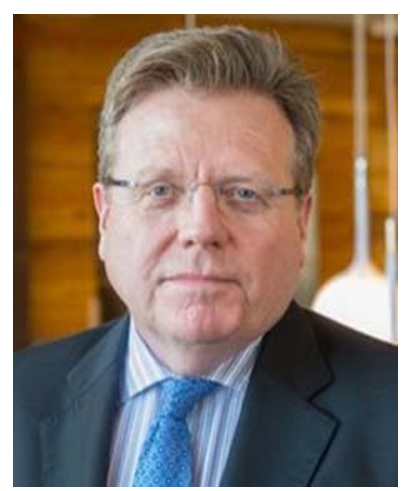

Matthew V. Tirrell 's research has been in the fields of polymer interfaces, dynamics, fluid phase behavior, and nanomedicine. He is particularly known for his work on polymer brushes, surface force measurement, peptide amphiphiles, and polyelectrolyte complex phase behavior. In 2011, Matthew Tirrell was appointed as the founding Pritzker Director and Dean of the Faculty of the Institute for Molecular Engineering and established the first University of Chicago engineering program, which he continues to oversee (now the Pritzker School of Molecular Engineering). Professor Tirrell simultaneously served as Deputy Laboratory Director for Science (September 2015-April 2018) and Chief Research Officer (January 2017-March 2018) at Argonne National Laboratory. Immediately prior to joining the University of Chicago, he was the Arnold and Barbara Silverman Professor and Chair of Bioengineering at the University of California, Berkeley, with additional appointments in chemical engineering and materials science and engineering, as well as a Faculty Scientist appointment at the Lawrence Berkeley National Laboratory. Dr. Tirrell completed 10 years as Dean of Engineering at the University of California, Santa Barbara, on June 30, 2009. From 1977 to 1999, he was on the faculty of Chemical Engineering and Materials Science at the University of Minnesota, where he served as department head from 1994 to 1999. Tirrell received a B.S. in Chemical Engineering at Northwestern University in 1973 and a Ph.D. in 1977 in Polymer Science from the University of Massachusetts. He has coauthored about 400 papers and one book, and has supervised about 100 Ph.D. students and 50 postdoctoral researchers. Professor Tirrell is a member of the National Academy of Engineering, the National Academy of Sciences, the American Academy of Arts and Sciences, and the Indian National Academy of Engineering, and is a Fellow of the American Institute of Medical and Biological Engineers, the AAAS, and the American Physical Society. It is a pleasure to make this contribution to a journal issue recognizing the many contributions of Matthias Ballauff to the science of macromolecules. Professor Ballauff has been a friend, scientific collaborator, and valued colleague for more than 30 years. All of his colleagues have enjoyed and learned from our associations with him. 